\title{
Distinct roles of VEGF-A and VEGF-C in tumour metastasis of gastric carcinoma
}

\author{
SHIGANG DING ${ }^{1,2}$, CHENGGANG LI ${ }^{2}$, SANREN LIN ${ }^{1}$, YAJING HAN ${ }^{1}$, \\ YU YANG ${ }^{3}$, YING ZHANG ${ }^{1}$, LINA LI ${ }^{1}$, LIYA ZHOU ${ }^{1}$ and SHANT KUMAR ${ }^{2}$ \\ ${ }^{1}$ Department of Gastroenterology, Peking University Third Hospital, Beijing 100083, P.R. China; ${ }^{2}$ Department \\ of Pathology, Medical School and Christie Hospital, The University of Manchester, Manchester M13 9PT, UK; \\ ${ }^{3}$ Department of Pathology, Peking University Health Science Centre, Beijing 100083, P.R. China
}

Received August 18, 2006; Accepted September 23, 2006

\begin{abstract}
The expression of VEGF-A and -C was investigated immunohistochemically in 51 specimens of gastric carcinoma. The expression intensity was evaluated by means of a semiquantitative scoring system and a quantitative analysis. The results showed that VEGF-A and $-\mathrm{C}$ were located mainly in the cytoplasm of tumour cells: $68.6 \%(34 / 51)$ and $62.8 \%$ (32/51) positive for VEGF-A and VEGF-C. VEGF-C expression was more frequent in tumours of the deceased patients $[88.2 \%(15 / 17)]$ compared to that in the survivors $[47.0 \%$ (16/34)]. Quantitative VEGF-C levels in the deceased patients were $0.304 \pm 0.023$, which was significantly elevated compared to that in the survivors $(0.131 \pm 0.018)(\mathrm{p}<0.01)$. The frequency of VEGF-C expression was higher in patients with lymph node metastases $(80.0 \%, 28 / 35)$ compared to those with negative nodes $(25 \%, 4 / 16)$. Accordingly, its levels were markedly elevated in the former $(0.230 \pm 0.017)$ than in the latter $(0.089 \pm 0.011)(\mathrm{p}<0.01)$. Furthermore, tumour cell invasion into the enlarged lymphatic vessels was also associated with VEGF-C expression. Its expression levels were $0.271 \pm 0.028$ in lymph vessel invasion and $0.153 \pm 0.021$ in the absence of invasion $(\mathrm{p}<0.01)$. VEGF-A expression, although correlated with survival, distant metastasis, ascites formation, and blood vessel invasion, was not associate with lymph node metastasis, lymphatic vessel invasion. The expression levels of the two factors were inversely correlated with overall survival rates. It can be conclude that VEGF-C expression in tumour tissues is indicative of lymphatic metastasis, whereas VEGF-A expression is more likely to be associated with haematogenous metastasis. Both VEGF-A and -C could serve as a prognostic biomarker in patients with gastric carcinoma.
\end{abstract}

Correspondence to: Professor Shant Kumar, Department of Pathology, Medical School, Stopford Building, University of Manchester, Manchester M13 9PT, UK

E-mail: shant.kumar@manchester.ac.uk or Professor Shigang Ding, Gastroenterology, Peking University, Beijing 100083, China E-mail: dingshigang222@163.com

Key words: gastric carcinoma, VEGF-A, VEGF-C, lymphangiogenesis, angiogenesis

\section{Introduction}

The major cause of cancer mortality is metastasis of tumour cells that can occur via the lymphatic vasculature and the blood vessels. Recent studies have indicated that lymphangiogenesis, the growth of lymphatic vessels, in the vicinity of solid tumours correlates with lymphatic spread (1-3). The principal growth factors and receptors for lymphangiogenesis have been identified as vascular endothelial growth factor $\mathrm{C}$ (VEGF-C), VEGF-D and VEGF receptor-3 (VEGFR-3) (4). Evidence from animal models and clinicopathological investigations in man has linked these signaling molecules to tumour lymphangiogenesis and lymphatic metastasis $(3,5,6)$. The importance of angiogenesis in tumour biology has been welldocumented (7). Angiogenesis is the driving force for tumour growth and metastasis. As a result, higher angiogenic activity in solid tumours is an indicator of metastatic potential and poor prognosis. Amongst growth factors, VEGF-A is considered as the most potent factor in angiogenesis, and its expression is correlated with tumour metastasis and prognosis (8).

VEGF-C, an VEGF isoform closely related to VEGF-D, preferentially binds to and signals via VEGFR-3 (6). VEGFR-3 is restricted to the venous endothelium during early stages of development and to the lymphatic endothelium in late stages (9). In animal models, VEGF-C induces lymphangiogenesis and lymphatic vessel enlargement $(10,11)$. VEGF-C also binds to VEGFR-2 of vascular endothelium. Thus it has an effect on angiogenesis as well (12). Compared with VEGF-A, VEGF-C is 4-5 times less potent on vascular permeability (13). Most studies have shown a positive correlation between the expression of VEGF-C and a range of clinicopathological features which reflect the prognosis of the patients (3). But discrepancy exists, as in some studies VEGF-C failed to be a prognostic factor, for instance, in colorectal cancer (14).

Angiogenesis plays a critical role in tumour progression. In the vast majority of malignancies, including gastric carcinoma, increased angiogenesis in the primary tumour has been associated with poor prognosis and relapse of the disease (8). One of the major factors regulating tumour angiogenesis is VEGF-A. Its expression in tumour tissues has been linked with metastasis and survival (15). VEGF-A is also a strong inducer of vascular permeability, which contributes to 
the formation of ascites, as well as plural effusion in peritoneal or thoracic malignancies (16).

Most investigations in tumours are focused on either VEGF-A or VEGF-C, whereas comparative studies on VEGF-A and VEGF-C in tumour progression are few. The current study has analysed the expression of both VEGF-A and VEGF-C in the same cohort of patients with gastric carcinoma, which has enabled us to simultaneously evaluate the roles of the two growth factors in tumour metastasis and prognosis.

\section{Materials and methods}

Clinical features of the patients. Ethics approval and informed consent from the patients were obtained for this study. Fiftyone tissue specimens were studied from patients with gastric carcinoma who underwent surgery. Clinical details of the patients are presented in Table I. Among these patients, 41 were males and 10 females and 17 had died by the end of the study. Thirty-five patients were histologically diagnosed with lymph node metastases, 15 with lymphatic invasion and 11 had venous invasion.

Immunohistochemical staining of VEGF-A and VEGF-C. Formalin-fixed and paraffin-embedded tissues were cut into $5-\mu \mathrm{m}$ thick sections and mounted on polylysine-coated slides. Sections were deparaffinized by xylene and dehydrated by a graded series of ethanols. After quenching the endogenous peroxidase activity with $0.3 \% \mathrm{H}_{2} \mathrm{O}_{2}$ for $30 \mathrm{~min}$, slides were microwaved for $10 \mathrm{~min}$, then cooled to room temperature for $20 \mathrm{~min}$. Sections were treated for $30 \mathrm{~min}$ at room temperature with $5 \%$ bovine serum albumin to block non-specific staining. Duplicate sections were then incubated overnight with the primary specific biotinylated antibodies against VEGF-A (rabbit IgG, diluted to 1:100), VEGF-C (rabbit IgG, diluted to 1:100). Both antibodies were purchased from R\&D Systems (Abingdon, UK). Sections were incubated with HRP-conjugated streptavidin (1:100 dilution) for $20 \mathrm{~min}$. Antibody binding was visualized with 3,3-diaminobenzidine tetrahydrochloride. Finally sections were lightly counterstained with Mayer's haematoxylin. Sections of resected colon tumours known to express VEGF-A and VEGF-C were used as positive controls and those incubated with normal rabbit IgG served as negative controls. VEGF-A or C expression was considered to be positive when $\geq 5 \%$ unequivocal cytoplasmic staining was seen in the tumour tissues.

Evaluation of the expression levels of VEGF-A, VEGF-C in the gastric carcinoma. The expression levels of VEGF-A and VEGF-C were assessed using two systems. One was the semi-quantitative scoring system by an experienced histopathologist blinded to the clinical information of the patients. The staining intensities of VEGF-A and VEGF-C were scored as: 0 , no staining; 1 , weak staining; 2 , moderate staining; 3 , strong staining. In each section, 10 high power fields of representative areas were counted at $\mathrm{x} 400$ magnification.

The staining intensities were also quantified using the computerised image analysis. Briefly, the immunostained sections were placed under a Leica (Leitz Laborlux S; Leica, Wetzlar, Germany) microscope coupled to a video camera
Table I. Clinical details of the 51 patients with gastric carcinoma.

Gender

Female

Median age (range)

$66(21-82)$ years

Median follow-up time (range)

$1.5(0.3-5)$ years

Deaths

Survivors

TNM stages

T1 (submucosa) 7

T2 (muscularis propria) $\quad 24$

T3 (serosa) 7

T4 (invade adjacent organs) 13

N0 (node negative) 16

N1 (1-6 nodes) 28

N2 (7-15 nodes)

$\mathrm{M} 0$

M1

Tumour location

Cardia and fundus

Body

Antrum

Diffuse

Tumour grade

Well-differentiated

Moderately and poorly differentiated

Lymphatic vessel invasion

Yes

No

Blood vessel invasion

Yes

No

Ascites

Yes

No

Helicobacter pylori infection

Yes

No

(Sony Hyper HAD; colour CCD-IRIS/RGB; model DXC151AP), connected to a computer aided image analysis system (Multi-Sync 3V; model JC-1535VMR; NEC Corp., CA, USA). The microscopic images captured and digitalised on to the video screen were analysed using an image analysis software programme (Leica Q500MC; Microsoft Windows hosted image analysis system; Leica Australia P/L). Because of the hetero- 


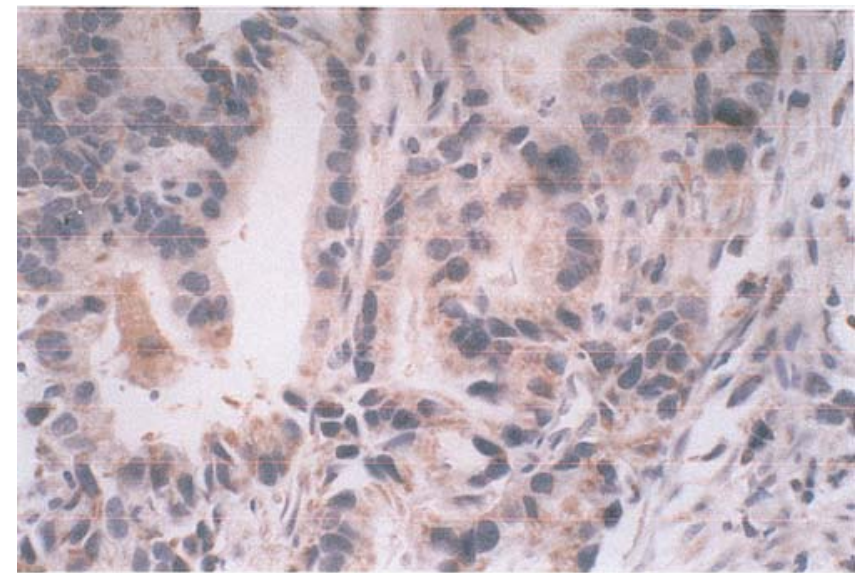

Figure 1. VEGF-A expression in gastric carcinoma. Immunohistochemical staining of cancer tissue showed that VEGF-A expression was located mainly in the cytoplasm of tumour cells, with minimal expression in spindle-like stromal cells (original magnification, $\mathrm{x} 400$ ).

geneity of the tumour tissues, 10 randomly selected fields were examined for each slide. The degree of staining intensity was calculated as the mean DAB stained area from a minimum of 800 cells in 10 microscopic fields at x400 magnification.

Statistical analysis. Data are presented as mean \pm SEM. Correlations between the semi-quantitative scores and the quantitative measurement of both VEGF-A and VEGF-C, expression levels with the clinicopathological features, were analysed by Spearman's correlation coefficient. Differences between groups were analysed using the non-parametric Mann-Whitney U test. Correlations between VEGF-A and VEGF-C with overall survival rates were determined by the Kaplan-Meier method. The mean values for VEGF-A (1.90) and VEGF-C (1.88) from the 51 patients were used as the cut-off values in the survival analysis. A p-value of $<0.05$ was considered statistically significant.

\section{Results}

Correlations between semi-quantitative scores and quantitative values of the staining intensity. The two procedures for VEGF-A ( $\mathrm{r}=0.946, \mathrm{p}=0.000)$ and VEGF-C measurement $(\mathrm{r}=0.936, \mathrm{p}=0.000)$, as analyzed by the Spearman's correlation coefficient, were well-correlated with each other.

Tissue expression of VEGF-C and VEGF-A. VEGF-A expression was observed in the cytoplasm of cancer cells and minimal in the stromal cells (Fig. 1), 68.6\% (34/51) of the carcinomas were VEGF-A positive. VEGF-C expression was restricted to the cytoplasm of cancer cells, with no expression in adjacent normal epithelial cells or stromal cells (Fig. 2). The percentage of VEGF-C positivity in gastric carcinoma was $62.8 \%(32 / 51)$. There was no correlation between VEGF-A and VEGF-C expression in cancer tissues $(r=0.138, p=0.335)$.

Correlations between VEGF-C expression and clinicopathological features. VEGF-C expression levels were divided into groups according to the clinicopathological features of the

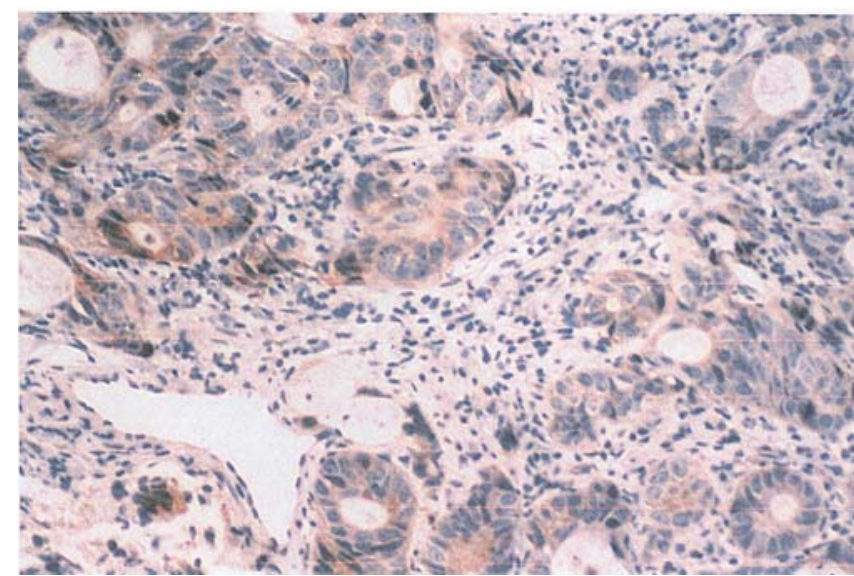

Figure 2. VEGF-C expression in gastric carcinoma. In this well-differentiated gastric carcinoma, VEGF-C expression was seen in the cytoplasm of tumour cells. Virtually all gland-like structures, composed of cancer cells, were positive for VEGF-C, in contrast to a clear background in stromal tissues (original magnification, x200).

patients. As presented in Fig. 3A, the 17 patients who died of the disease possessed the highest levels of VEGF-C $(0.304 \pm 0.023)$, which were significantly higher than those who had survived $(0.131 \pm 0.018)(\mathrm{p}<0.01)$. VEGF-C in cancer tissues was positive in $88.2 \%(15 / 17)$ deceased patients, in contrast to $47 \%(16 / 34)$ in survivors. VEGF-C expression was more frequently positive in patients with lymph node metastases $(28 / 35,80 \%)$ than those with no node metastases $(4 / 16,25 \%)$. In patients with lymph node metastasis, VEGF-C levels were also markedly elevated compared with those without node involvement $(0.230 \pm 0.017$ vs $0.089(0.011, \mathrm{p}<0.01)$. Furthermore, the expression of VEGF-C was related to lymphatic invasion. In 13 of the 32 patients with VEGF-C positivity $(40.6 \%)$, tumour cells were present within the enlarged lymphatics. VEGF-C levels were higher in patients with lymphatic vessel invasion than in those without lymphatic vessel invasion $(0.271 \pm 0.028$ vs $0.153 \pm 0.021$; $\mathrm{p}<0.01)$. However, VEGF-C levels were not correlated with blood vessel invasion (blood vessel involvement: $0.247 \pm 0.040$ vs no blood vessel involvement: $0.172 \pm 0.020 ; \mathrm{p}>0.05$ ) (Fig. 4A). With regard to association of VEGF-C with depth of tumour invasion, increased VEGF-C levels were observed in tumours invading the serosa $(0.307 \pm 0.054 ; \mathrm{p}<0.05$ compared with the submucosa, $0.144 \pm 0.053$; muscularis propria, $0.167 \pm 0.021$; or adjacent organs, $0.195 \pm 0.041)$. Further analysis found no correlations of VEGF-C levels with distant metastases, ascites, Helicobacter pylori infection, age, gender, location of the tumour or histopathological grade.

Correlations between VEGF-A expression and the clinicopathological features. VEGF-A expression levels were divided into groups in the same way as for VEGF-C. VEGF-A was more frequently observed in deceased patients $(14 / 17$, $82.4 \%)$, compared with those who had survived (19/32, $59.4 \%$ ). VEGF-A expression levels in patients who had survived was lower than in those who had died of the disease $(0.155 \pm 0.020$ vs $0.273 \pm 0.027 ; \mathrm{p}<0.01)$ (Fig. 3B). Markedly elevated VEGF-A levels were also noted in patients with distant metastases over those without distant metastases 

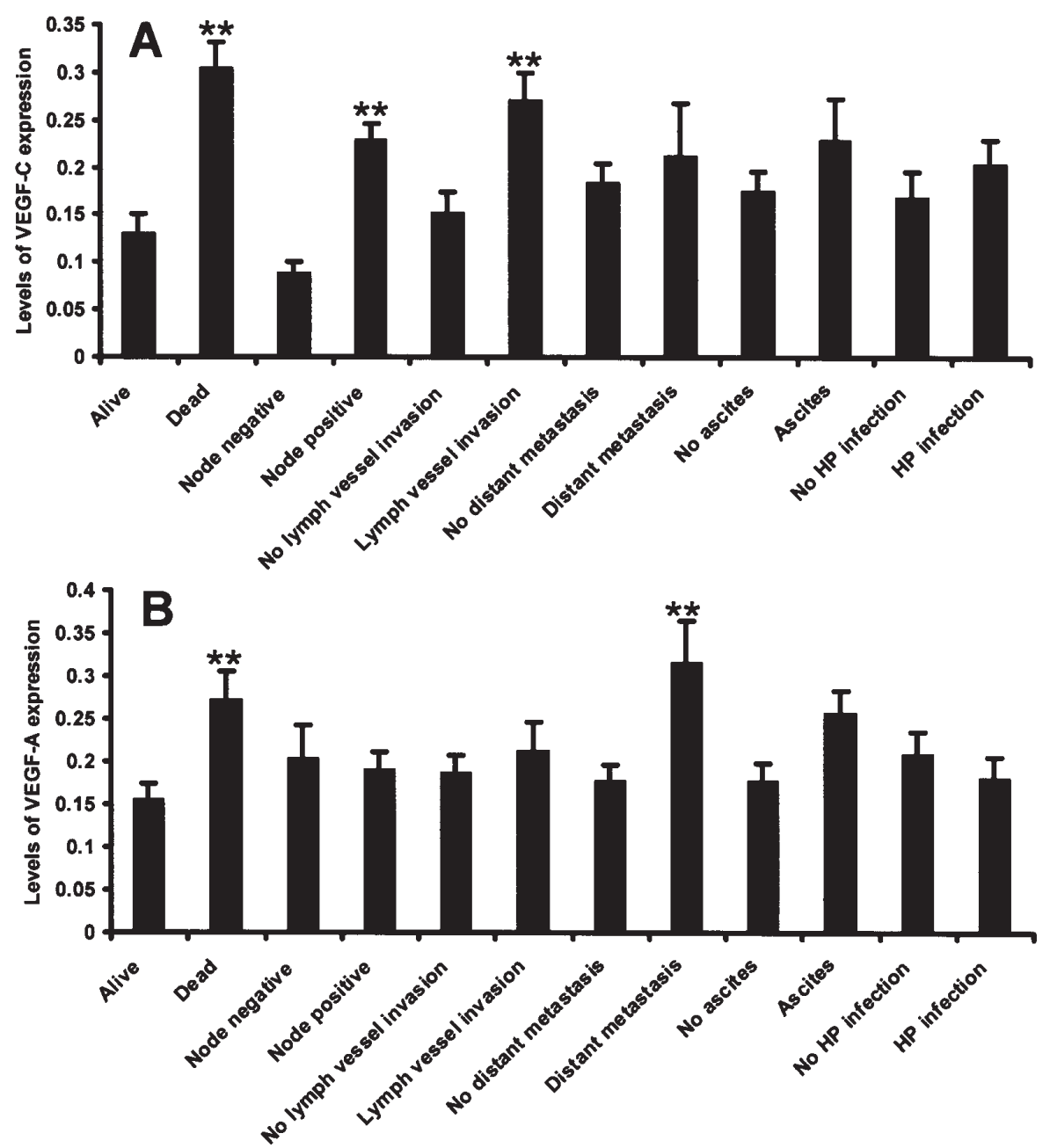

Figure 3. VEGF-A and VEGF-C expression in gastric carcinoma and their association with clinicopathological features. HP, helicobacter pylori. ${ }^{*} \mathrm{p}<0.05$ and ${ }^{* *} \mathrm{p}<0.01$, Mann-Whitney U test.
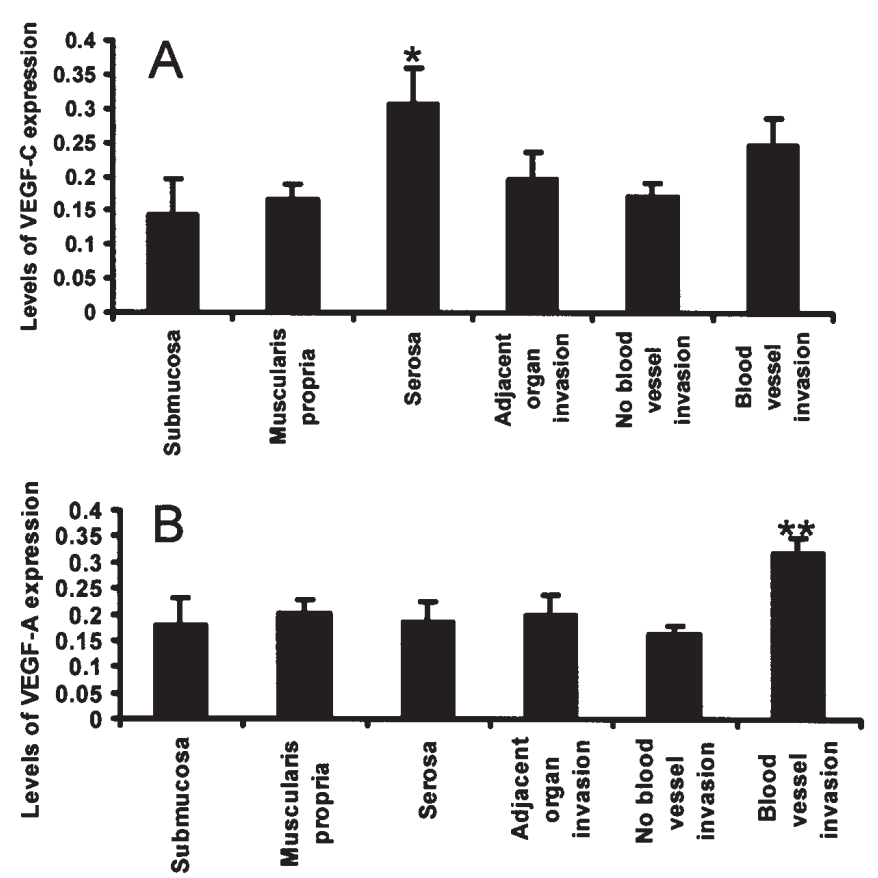

Figure 4. Correlation of VEGF-A and VEGF-C expression with the depth of tumour invasion. ${ }^{*} \mathrm{p}<0.05$ and $^{* *} \mathrm{p}<0.01$, Mann-Whitney U test.
(0.316 \pm 0.049 vs $0.178 \pm 0.018 ; \mathrm{p}<0.01)$. VEGF-A expression was significantly correlated with peritoneal dissemination; all of the 11 patients with ascites were positive for VEGF-A, in comparison to 23 of $40(57.5 \%)$ patients without ascites, but VEGF-A positive. The levels of VEGF-A in patients with ascites were higher than in those without it $(0.258 \pm 0.026$ vs $0.177 \pm 0.021 ; \mathrm{p}<0.05)$. There was no association between VEGF-A and the depth of invasion, whereas its levels were significantly increased in patients with blood vessel invasion, compared to those without blood vessel invasion $(0.318 \pm 0.031$ vs $0.161 \pm 0.018, \mathrm{p}<0.01)$ (Fig. 4B). Further analysis showed no correlation between VEGF-A levels and lymph node involvement, lymphatic vessel invasion, Helicobacter infection, age, gender, location of the tumour or histopathological grade.

VEGF-A and VEGF-C are both correlated with overall survival. At the end of this study, 17 of 51 patients had died of their disease. The associations between VEGF-A or VEGF-C expression and overall survival rates were evaluated using the Kaplan-Meier analysis. As shown in Fig. 5, VEGF-A expression was significantly associated with overall survival rates $(\mathrm{p}=0.001)$ - patients expressing VEGF-A $>1.90$ in the tumour had a strong probability of poor prognosis. Similarly 
A

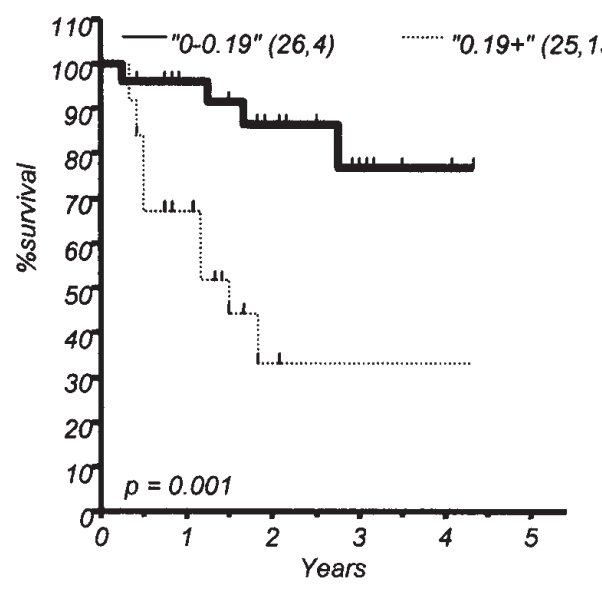

B

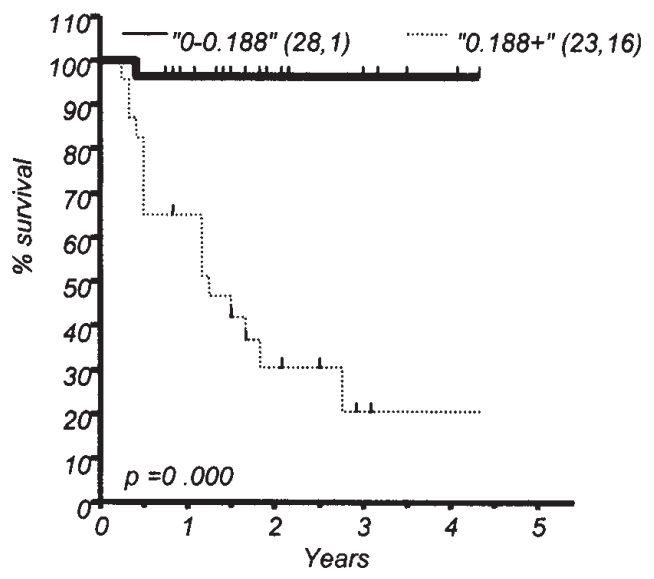

Figure 5. Kaplan-Meier analysis of correlation of VEGF-A and VEGF-C with overall survival rates. (A), Those patients expressing higher levels of VEGF-A (dotted line) were more likely to have a poorer prognosis than those expressing low levels of VEGF-A (solid line). (B), The probability of death from cancer was significantly increased in patients with high VEGF-C expression (dotted line) than those expressing low levels of VEGF-C in their tumours (solid line).

Table II. Correlation of VEGF-A and VEGF-C with clinicopathological parameters.

\begin{tabular}{lccccc}
\hline & \multicolumn{2}{c}{ VEGF-A } & & \multicolumn{2}{c}{ VEGF-C } \\
\cline { 2 - 3 } \cline { 6 - 6 } & $\mathrm{r}$ & $\mathrm{p}$ & $\mathrm{r}$ & $\mathrm{p}$ \\
\hline Age & 0.150 & 0.295 & & 0.045 & 0.754 \\
Gender & 0.044 & 0.761 & & 0.035 & 0.806 \\
Survival & -0.449 & $0.001^{\mathrm{b}}$ & & -0.619 & $0.000^{\mathrm{b}}$ \\
Node metastasis & 0.009 & 0.954 & & 0.732 & $0.000^{\mathrm{b}}$ \\
Lymphatic invasion & -0.076 & 0.596 & & 0.427 & $0.002^{\mathrm{b}}$ \\
Blood vessel invasion & 0.486 & $0.000^{\mathrm{b}}$ & & 0.227 & 0.110 \\
Tumour location & 0.060 & 0.677 & & 0.228 & 0.108 \\
Histological grade & 0.240 & 0.081 & & 0.120 & 0.390 \\
Ascites & 0.288 & $0.040^{\mathrm{a}}$ & & 0.155 & 0.276 \\
HP infection & 0.097 & 0.496 & & 0.140 & 0.327 \\
TNM stage & & & & \\
T & 0.022 & 0.878 & 0.275 & $0.049^{\mathrm{a}}$ \\
$\mathrm{N}$ & 0.078 & 0.587 & 0.560 & $0.000^{\mathrm{b}}$ \\
M & 0.341 & $0.014^{\mathrm{a}}$ & 0.068 & 0.634 \\
\hline
\end{tabular}

${ }^{\mathrm{a}} \mathrm{p}<0.05$ and ${ }^{\mathrm{b}} \mathrm{p}<0.01$, Spearman's correlation coefficient.

to VEGF-A, VEGF-C expression was correlated with overall survival rates $(\mathrm{p}=0.000)$. The probability of death from cancer was remarkably higher in patients with VEGF-C $>0.188$ in their tumour tissues (Table II).

\section{Discussion}

Angiogenesis and lymphangiogenesis are two distinct concepts in tumour biology. Both critically influence tumour growth and spread. The identification of VEGF-A and VEGF-C and their cognate cell surface receptors, which control the formation of blood vessels and lymphatics, has greatly advanced our understanding of these complex processes. VEGF-A is regarded as the most potent angiogenic factor whereas VEGF-C is considered to be the driver for lymphangiogenesis. Tumour spread via lymphatic and haematogenous routes is the most common cause for cancer deaths where VEGF-A and VEGF-C are involved. In the present study, we have investigated the expression levels of VEGF-A and VEGF-C in gastric carcinoma and evaluated their association with tumour spread and prognosis. The data showed that although the expression levels of the two factors were inversely correlated with survival, they had dissimilar roles in tumour metastasis. While VEGF-A was positively correlated with distant metastasis and venous invasion, VEGF-C was strongly correlated with lymph node metastasis, lymphatic vessel invasion and the depth of tumour invasion, indicating their distinct functions in the progression of gastric carcinoma.

The lymphatic vessels are major routes for cancer cell metastasis to the lymph nodes. The presence of tumour cells in the local lymph nodes is significant for the staging of cancer and hence it correlates well with prognosis. Lymphatic metastasis had been long considered to be a passive process whereby detached tumour cells implanted in the lymph nodes via drainage though pre-existing local lymphatic vessels. Recent evidence suggests that lymphangiogenesis can contribute actively to metastasis. In animal models of breast cancer, VEGF-C promoted growth of tumour-associated lymphatics infiltrated with tumour cells (17). Similarly, VEGF-C induced intratumoural lymphangiogenesis, when expressed in human breast cancer cells orthotopically 
transplanted onto nude mice, that led to enhanced metastasis to regional lymph nodes and to the lung (18). Some clinicopathological studies showed that VEGF-C expression was closely related to lymphatic invasion and lymph node involvement in carcinomas of the lung, breast, thyroid, prostate, colon, rectum and oesophagus $(3,18,19)$. The present findings are entirely consistent with previous reports and they further confirm that VEGF-C expression is associated with the depth of invasion and survival. Therefore it is a prognostic factor in patients with gastric carcinoma.

The fact that tumour growth and metastasis depends on angiogenesis has been well-proven. Intratumoural microvessel density is an independent prognostic factor in a range of malignancies (20-22). Accordingly, the expression of several angiogenic factors and their cognate receptors in tumours is indicative of metastasis and prognosis (21). The growth and maturation of tumour neovasculature are finely regulated and complex processes involving numerous angiogenic and antiangiogenic factors produced by tumour and stromal cells. VEGF-A is the best known and potent angiogenic factor that signals to its target endothelial cells predominantly via VEGFR-1 and VEGFR-2 (23). It stimulates neovascularisation by promoting endothelial cell proliferation, migration and survival (23). Consequently, the expression of VEGF-A has been reported to be increased in gastric carcinoma and correlates with disease recurrence and survival (24). In this study, VEGF-A expression was correlated with distant metastasis, blood vessel invasion and survival. However, no significant correlation was shown with the depth of invasion or lymph node metastasis. These findings highlight the involvement of VEGF-A in haematogenous metastasis of gastric carcinoma. VEGF-A was originally discovered as a vascular permeability factor and plays an important role in malignant ascites formation $(16,25)$. Animal models have suggested that the mean volume of ascites, number of tumour cells in the ascites, and the peritoneal capillary permeability are suppressed with anti-VEGF-A neutralizing antibody (26), providing evidence for its critical role in ascites formation. The correlation of VEGF-A with the presence of ascites in patients with gastric carcinoma confirms the data from the animal study.

Angiogenesis and lymphangiogenesis, although both contribute to tumour progression, are two different overlapping pathological processes in malignancies. The formation of extensive microvessels within the tumour provides nutrients and facilitates tumour cell invasion into the blood stream, thus increasing the risk of distant metastasis and a poorer prognosis. In contrast, the lymphatic system drains interstitial fluids from the tumour to the sentinel lymph nodes. Therefore, active lymphatic vessel formation in the tumour may lead to lymphatic dissemination. The data from this study have revealed the distinct roles of VEGF-A and VEGF-C in gastric carcinoma. A heightened production of VEGF-A by tumour tissues makes a tumour liable to haematogenous spread, whereas increased VEGF-C in a tumour is a risk factor for lymphatic metastasis. Therefore, simultaneous detection of VEGF-A and VEGF-C is necessary for predicting tumour progression in gastric carcinoma.

In summary, we have shown that tumours expressing VEGF-C are more inclined to lymph node metastasis and lymphatic invasion. In comparison, elevated tissue expression of VEGF-A is correlated with blood vessel invasion, distant metastasis and ascites formation. The expression levels of VEGF-A and VEGF-C in the tumour tissues were inversely correlated with overall survival rates. This study has demonstrated distinct roles for these two growth factors in the pathobiology of gastric carcinoma and suggests that both can serve as prognostic factors in assessing prognosis for patients with gastric carcinoma.

\section{References}

1. Achen MG, McColl BK and Stacker SA: Focus on lymphangiogenesis in tumor metastasis. Cancer Cell 7: 121-127, 2005.

2. Duff SE, Li C, Jeziorska M, Kumar S, Saunders MP, Sherlock D, O'Dwyer ST and Jayson GC: Vascular endothelial growth factors $\mathrm{C}$ and $\mathrm{D}$ and lymphangiogenesis in gastrointestinal tract malignancy. Br J Cancer 89: 426-430, 2003.

3. Stacker SA, Williams RA and Achen MG: Lymphangiogenic growth factors as markers of tumor metastasis. APMIS 112: 539-549, 2004.

4. Makinen T and Alitalo K: Molecular mechanisms of lymphangiogenesis. Cold Spring Harb Symp Quant Biol 67: 189-196, 2002.

5. He Y, Karpanen $\mathrm{T}$ and Alitalo K: Role of lymphangiogenic factors in tumor metastasis. Biochim Biophys Acta 1654: 3-12, 2004.

6. Saharinen P, Tammela T, Karkkainen MJ and Alitalo K: Lymphatic vasculature: development, molecular regulation and role in tumor metastasis and inflammation. Trends Immunol 25: 387-395, 2004.

7. Folkman J: Angiogenesis in cancer, vascular, rheumatoid and other disease. Nat Med 1: 27-31, 1995.

8. Chung YS, Maeda K and Sowa M: Prognostic value of angiogenesis in gastro-intestinal tumours. Eur J Cancer 32A: 2501-2505, 1996.

9. Tammela T, Enholm B, Alitalo K and Paavonen K: The biology of vascular endothelial growth factors. Cardiovasc Res 65: 550-563, 2005.

10. Enholm B, Karpanen T, Jeltsch M, Kubo H, Stenback F, Prevo R, Jackson DG, Yla-Herttuala S and Alitalo K: Adenoviral expression of vascular endothelial growth factor-C induces lymphangiogenesis in the skin. Circ Res 88: 623-629, 2001.

11. Jeltsch M, Kaipainen A, Joukov V, Meng X, Lakso M, Rauvala H, Swartz M, Fukumura D, Jain RK and Alitalo K: Hyperplasia of lymphatic vessels in VEGF-C transgenic mice. Science 276: 1423-1425, 1997.

12. Cao Y, Linden P, Farnebo J, Cao R, Eriksson A, Kumar V, Qi JH, Claesson-Welsh L and Alitalo K: Vascular endothelial growth factor $\mathrm{C}$ induces angiogenesis in vivo. Proc Natl Acad Sci USA 95: 14389-14394, 1998 .

13. Joukov V, Sorsa T, Kumar V, Jeltsch M, Claesson-Welsh L, Cao Y, Saksela O, Kalkkinen N and Alitalo K: Proteolytic processing regulates receptor specificity and activity of VEGF-C. EMBO J 16: 3898-3911, 1997.

14. George ML, Tutton MG, Janssen F, Arnaout A, Abulafi AM, Eccles SA and Swift RI: VEGF-A, VEGF-C, and VEGF-D in colorectal cancer progression. Neoplasia 3: 420-427, 2001.

15. Hicklin DJ and Ellis LM: Role of the vascular endothelial growth factor pathway in tumor growth and angiogenesis. J Clin Oncol 23: 1011-1027, 2005.

16. Zebrowski BK, Liu W, Ramirez K, Akagi Y, Mills GB and Ellis LM: Markedly elevated levels of vascular endothelial growth factor in malignant ascites. Ann Surg Oncol 6: 373-378, 1999.

17. Karpanen T, Egeblad M, Karkkainen MJ, Kubo H, YlaHerttuala S, Jaattela M and Alitalo K: Vascular endothelial growth factor $\mathrm{C}$ promotes tumor lymphangiogenesis and intralymphatic tumor growth. Cancer Res 61: 1786-1790, 2001.

18. Skobe M, Hawighorst T, Jackson DG, Prevo R, Janes L, Velasco P, Riccardi L, Alitalo K, Claffey K and Detmar M: Induction of tumor lymphangiogenesis by VEGF-C promotes breast cancer metastasis. Nat Med 7: 192-198, 2001.

19. Nathanson SD: Insights into the mechanisms of lymph node metastasis. Cancer 98: 413-423, 2003.

20. Duff SE, Li C, Garland JM and Kumar S: CD105 is important for angiogenesis: evidence and potential applications. FASEB J 17: 984-992, 2003. 
21. Ellis LM: Angiogenesis and its role in colorectal tumor and metastasis formation. Semin Oncol 31: 3-9, 2004.

22. Kumar S, Ghellal A, Li C, Byrne G, Haboubi N, Wang JM and Bundred N: Breast carcinoma: vascular density determined using CD105 antibody correlates with tumor prognosis. Cancer Res 59: 856-861, 1999.

23. Zachary I and Gliki G: Signaling transduction mechanisms mediating biological actions of the vascular endothelial growth factor family. Cardiovasc Res 49: 568-581, 2001.

24. Yao JC, Wang L, Wei D, Gong W, Hassan M, Wu TT, Mansfield P, Ajani J and Xie K: Association between expression of transcription factor $\mathrm{Sp} 1$ and increased vascular endothelial growth factor expression, advanced stage, and poor survival in patients with resected gastric carcinoma. Clin Cancer Res 10: 4109-4117, 2004.
25. Verheul HM, Hoekman K, Jorna AS, Smit EF and Pinedo HM: Targeting vascular endothelial growth factor blockade: ascites and pleural effusion formation. Oncologist 5: 50, 2000.

26. Yoshiji H, Kuriyama S, Hicklin DJ, Huber J, Yoshii J, Ikenaka Y, Noguchi R, Nakatani T, Tsujinoue $\mathrm{H}$ and Fukui H: The vascular endothelial growth factor receptor KDR/Flk-1 is a major regulator of malignant ascites formation in the mouse hepatocellular carcinoma model. Hepatology 33: 841-847, 2001. 